\title{
PATTERN INTEGRATION WITH IMPROPER RIEMANN INTEGRALS
}

\author{
R. E. CARR
}

1. Introduction. In a previous paper $[1]^{1}$ we have recently introduced and discussed a type of integration, which we have called pattern integration, and have established the following theorem:

Theorem 1.1. Let $f(x)$ be (proper) Riemann integrable, $0 \leqq x \leqq 1$. Let $(k-1) / n \leqq \xi_{k}^{(n)} \leqq k / n(k=1,2,3, \cdots, n)$. Let the pattern $P$ be characterized by the dyadic number

$$
t=0 . \alpha_{1} \alpha_{2} \alpha_{3} \cdots \alpha_{n} \cdots(2)
$$

such that

$$
\lim _{n \rightarrow \infty} \frac{1}{n} \sum_{k=1}^{n} \alpha_{k}=\alpha
$$

Then the pattern integral

$$
\text { (P) } \int_{0}^{1} f(x) d x \equiv \lim _{n \rightarrow \infty} \frac{1}{n} \sum_{k=1}^{n} \alpha_{k} f\left(\xi_{k}^{(n)}\right)=\alpha(R) \int_{0}^{1} f(x) d x .
$$

It seems desirable to extend this result, if possible, to improper Riemann integrals. In this paper we shall confine our remarks to improper integrals of the form

$$
\oint_{0}^{1} f(x) d x \equiv \lim _{\epsilon \rightarrow 0+}(R) \int_{\epsilon}^{1} f(x) d x
$$

$(R)$ in front of any integral will indicate a proper Riemann integral.

2. The improper integral defined as the limit of a sum. We first consider the case where $\alpha_{k}=1(k=1,2,3, \cdots)$. A fundamental difficulty not encountered in the previous paper is apparent from the definition of $\Phi_{0}^{1} f(x) d x$. Bromwich and Hardy have considered this problem [2]. As they point out, the ordinary definition of this improper integral is by means of a double (repeated) limit; and in order to replace this limit by the single limit,

$$
\lim _{n \rightarrow \infty} \frac{1}{n} \sum_{k=1}^{n} f\left(\xi_{k}^{(n)}\right)
$$

Received by the editors December 11, 1950.

${ }^{1}$ Numbers in brackets refer to the bibliography at the end of the paper. 
some restrictions are certainly necessary. In the first place, if $0<\xi_{1}^{(n)}$ $\leqq 1 / n$, we can clearly choose $\xi_{1}^{(n)}$ so that $\left|(1 / n) f\left(\xi_{1}^{(n)}\right)\right|$ is greater than any assigned number. It is then suggested that the most obvious choice is $\xi_{\mathbb{z}}^{(n)}=k / n$, and with this restriction they establish:

TheOREM 2.1. Let $f(x)$ be positive and tend steadily to $\infty$ as $x$ tends to zero. Let $\oint_{0}^{1} f(x) d x$ be convergent. Then

$$
\lim _{n \rightarrow \infty} \frac{1}{n} \sum_{k=1}^{n} f\left(\frac{k}{n}\right)=\oint_{0}^{1} f(x) d x .
$$

The proof rests on the fact that for all $n$,

$$
\frac{1}{n} \sum_{k=2}^{n} f\left(\frac{k}{n}\right) \leqq(R) \int_{1 / n}^{1} f(x) d x \leqq \frac{1}{n} \sum_{k=1}^{n-1} f\left(\frac{k}{n}\right),
$$

while the difference between the two sums in (2.1) is

$$
\frac{1}{n}\left\{f\left(\frac{1}{n}\right)-f(1)\right\} \text {. }
$$

That the limit of (2.2), as $n \rightarrow \infty$, is zero, follows from the well known result that if $f(x)$ steadily increases as $x$ decreases and $\oint_{0}^{1} f(x) d x$ is convergent, then $\lim _{x \rightarrow 0+} x f(x)=0$. From this the conclusion is immediate.

It appears that the following extension of the above theorem is new, although the theorem is quite elementary.

TheOREM 2.2. Let $f(x)$ be positive and tend steadily to $\infty$ as $x$ tends to zero. Let $\oint_{0}^{1} f(x) d x$ be convergent. Let $(1 / M n) \leqq \xi_{1}^{(n)} \leqq(1 / n) \quad(M \geqq 1$ and fixed), and $(k-1) / n \leqq \xi_{\mathfrak{k}}^{(n)} \leqq(k / n) \quad(k=2,3, \cdots, n)$. Then

$$
\lim _{n \rightarrow \infty} \frac{1}{n} \sum_{k=1}^{n} f\left(\xi_{k}^{(n)}\right)=\oint_{0}^{1} f(x) d x \text {. }
$$

Proof. Define

$$
A_{n} \equiv \frac{1}{n} \sum_{k=1}^{n} f\left(\frac{k}{n}\right)
$$

and

$$
\begin{aligned}
B_{n} & \equiv \frac{1}{n}\left\{f\left(\frac{1}{M n}\right)+\sum_{k=2}^{n} f\left(\frac{k-1}{n}\right)\right\} \\
& \equiv \frac{1}{n}\left\{f\left(\frac{1}{M n}\right)+\sum_{k=1}^{n} f\left(\frac{k}{n}\right)-f(1)\right\} .
\end{aligned}
$$


Then

$$
A_{n} \leqq \frac{1}{n} \sum_{k=1}^{n} f\left(\xi_{k}^{(n)}\right) \leqq B_{n} .
$$

But, clearly, $\lim _{n \rightarrow \infty} A_{n}=\lim _{n \rightarrow \infty} B_{n}=\oint_{0}^{1} f(x) d x$, and our conclusion follows.

A sharper criterion, in order that the relation

$$
\lim _{n \rightarrow \infty} \frac{1}{n} \sum_{k=1}^{n} f\left(\frac{k}{n}\right)=\oint_{0}^{1} f(x) d x
$$

should hold whenever the improper integral on the right is convergent, has been given by Wintner [3] in the following result:

THEOREM 2.3. If $f(x)$ is of bounded variation on every interval $\epsilon \leqq x \leqq 1$, where $\epsilon>0$, and behaves, as $x \rightarrow 0$, so as to satisfy the restriction

$$
\int_{e}^{1}|d f(x)|=o\left(\epsilon^{-1}\right),
$$

then the convergence of $\oint_{0}^{1} f(x) d x$ implies that

$$
\lim _{\epsilon \rightarrow 0+} \in \sum_{k \in \leqq 1} f(k \epsilon)
$$

exists and equals $\oint_{0}^{1} f(x) d x$.

The result (2.3) would follow from (2.4) by letting $\epsilon=1 / n$.

The following extension of Wintner's result appears to be new:

THEOREM 2.4. Let $f(x)$ be of bounded variation on every interval $\epsilon \leqq x \leqq 1$, where $\epsilon>0$, and let $f(x)$ behave, as $x \rightarrow 0^{+}$, so as to satisfy the restriction

$$
\int_{\epsilon}^{1}|d f(x)|=o\left(\epsilon^{-1}\right) .
$$

Let $\xi_{1}=1 / M n(M \geqq 1$ and fixed $)$, and $(k-1) / n \leqq \xi_{k}^{(n)} \leqq(k / n)(k=2,3$, $\cdots, n)$. Let $\oint_{0}^{1} f(x) d x$ exist.

Then

$$
\lim _{n \rightarrow \infty} \frac{1}{n} \sum_{k=1}^{n} f\left(\xi_{k}^{(n)}\right)=\oint_{0}^{1} f(x) d x .
$$

Proof. From the hypothesis it follows that

$$
\lim _{x \rightarrow 0} x f(x)=0 \text {. }
$$


For,

$$
\epsilon|f(1)-f(\epsilon)|=\epsilon\left|\int_{\epsilon}^{1} d f(x)\right| \leqq \epsilon \int_{\epsilon}^{1}|d f(x)| \rightarrow 0 .
$$

From Wintner's result, we know

$$
\lim _{n \rightarrow \infty} \frac{1}{n} \sum_{k=1}^{n} f\left(\frac{k}{n}\right)=\oint_{0}^{1} f(x) d x .
$$

Consider

$$
\left\{\frac{1}{n} \sum_{k=1}^{n} f\left(\xi_{k}^{(n)}\right)-\frac{1}{n} \sum_{k=1}^{n} f\left(\frac{k}{n}\right)\right\}
$$

For all $n$,

$$
\begin{aligned}
\left|\sum_{k=1}^{n} f\left(\xi_{k}^{(n)}\right)-\sum_{k=1}^{n} f\left(\frac{k}{n}\right)\right| & \leqq \sum_{k=1}^{n}\left|f\left(\xi_{k}^{(n)}\right)-f\left(\frac{k}{n}\right)\right| \\
& <\int_{(1 / n)}^{1}|d f(x)|+\left|f\left(\frac{1}{M n}\right)\right|+\left|f\left(\frac{1}{n}\right)\right| .
\end{aligned}
$$

Hence, using (2.5) and (2.6),

$$
\lim _{n \rightarrow \infty}\left|\frac{1}{n} \sum_{k=1}^{n} f\left(\xi_{k}^{(n)}\right)-\frac{1}{n} \sum_{k=1}^{n} f\left(\frac{k}{n}\right)\right|=0,
$$

and our conclusion follows.

It should be noted that a number of theorems are available in the "converse" direction, that is, in which the convergence of the improper integral $\mathscr{S}_{0}^{1} f(x) d x$ follows from the knowledge of the existence of $\lim _{\epsilon \rightarrow 0+} \epsilon \sum_{n \epsilon \leqq 1} f(n \epsilon)$. In this paper, however, we are not concerned with such results.

3. A pattern integral theorem. To hope for a complete extension of Theorem 1.1 to improper integrals of the form $\mathscr{\Phi}_{0}^{1} f(x) d x$ is too much. Counter examples are not difficult to construct. For example, see $\$ 5$ of the paper by Bromwich and Hardy [2].

The following theorem partially extends the earlier results.

TheOREM 3.1. Let $f(x)$ be positive and tend steadily to $\infty$ as $x$ tends to zero. Let $\Phi_{0}^{1} f(x) d x$ be convergent. Let $(1 / M n) \leqq \xi_{1}^{(n)} \leqq(1 / n)(M \geqq 1$ and fixed $)$, and $(k-1) / n \leqq \xi_{k}^{(n)} \leqq k / n(k=2,3, \cdots, n)$. Let the pattern 
$P$ be characterized by the dyadic number

$$
t=0 . \alpha_{1} \alpha_{2} \alpha_{3} \cdots \alpha_{n} \cdots(2),
$$

such that

$$
\lim _{n \rightarrow \infty} \frac{1}{n} \sum_{k=1}^{n} \alpha_{k}=\alpha
$$

Then

$$
\text { (P) } \oint_{0}^{1} f(x) d x \equiv \lim _{n \rightarrow \infty} \frac{1}{n} \sum_{k=1}^{n} \alpha_{k} f\left(\xi_{k}^{(n)}\right)=\alpha \oint_{0}^{1} f(x) d x .
$$

Proof. Define $f_{N}(x)=f(1 / M N), 0 \leqq x<1 / N, f_{N}(x)=f(x), 1 / N$ $\leqq x \leqq 1$. For all values of $N>0,(R) \int_{0}^{1} f_{N}(x) d x$ exists and

$$
\text { (P) } \int_{0}^{1} f_{N}(x) d x=\alpha(R) \int_{0}^{1} f_{N}(x) d x .
$$

Consider the expression

$$
\frac{1}{n} \sum_{k=1}^{n} \alpha_{k} f\left(\xi_{k}^{(n)}\right)
$$

Let the subinterval in which $1 / N$ occurs be the $\lambda(n)$ th. (Then $\lim _{n \rightarrow \infty} \lambda(n) / n=1 / N$.) Hence (3.2) can be written as $\mathrm{I}_{n}+\mathrm{II}_{n}-\mathrm{III}_{n}$ $+\mathrm{IV}_{n}$, where

$$
\begin{aligned}
\mathrm{I}_{n} & =\frac{1}{n} \alpha_{\lambda(n)} f\left(\xi_{\lambda(n)}^{(n)}\right), \quad \mathrm{II}_{n}=\frac{1}{n} \sum_{k=1}^{n} \alpha_{k} f_{N}\left(\xi_{k}^{(n)}\right), \\
\mathrm{III}_{n} & =\frac{1}{n} \sum_{k=1}^{\lambda(n)} \alpha_{k} f_{N}\left(\xi_{k}^{(n)}\right), \quad \mathrm{IV}_{n}=\frac{1}{n} \sum_{h=1}^{\lambda(n)-1} \alpha_{k} f\left(\xi_{k}^{(n)}\right) .
\end{aligned}
$$

Let us consider the behavior of $\mathrm{I}_{n}, \mathrm{II}_{n}, \mathrm{III}_{n}$, and $\mathrm{IV}_{n}$, as $n \rightarrow \infty$. Clearly $f\left(\xi_{\lambda(n)}^{(n)}\right) \leqq f(1 / M n)$, and since $\lim _{x \rightarrow 0+} x f(x)=0, \lim _{n \rightarrow \infty} \mathrm{I}_{n}=0$. From (3.1), we see that

$$
\lim _{n \rightarrow \infty} \mathrm{II}_{n}=\alpha(R) \int_{0}^{1} f_{N}(x) d x=\alpha\left\{\frac{1}{N} f\left(\frac{1}{M N}\right)+(R) \int_{1 / N}^{1} f(x) d x\right\} .
$$

Now

$$
\mathrm{III}_{n}=f\left(\frac{1}{M N}\right) \frac{\lambda(n)-1}{n} \frac{1}{\lambda(n)-1} \sum_{k=1}^{\lambda(n)-1} \alpha_{k}+\frac{1}{n} \alpha_{\lambda(n)} f_{N}\left(\xi_{\lambda(n)}^{(n)}\right) .
$$

Hence, 


$$
\lim _{n \rightarrow \infty} \operatorname{III}_{n}=\alpha \frac{1}{N} f\left(\frac{1}{M N}\right)
$$

We have

$$
\mathrm{IV}_{n} \leqq \frac{1}{n} \sum_{k=1}^{\lambda(n)-1} f\left(\xi_{k}^{(n)}\right)
$$

Hence

$$
0 \leqq \mathrm{IV}_{n} \leqq \oint_{0}^{1 / N} f(x) d x+\frac{1}{n} f\left(\frac{1}{M n}\right)
$$

for all $n$. We then see that, as $n \rightarrow \infty$, the expression (3.2) at worst oscillates between

$$
\alpha(R) \int_{1 / N}^{1} f(x) d x
$$

and

$$
\alpha(R) \int_{1 / N}^{1} f(x) d x+\oint_{0}^{1 / N} f(x) d x .
$$

But this is true for all $N>0$, so letting $N \rightarrow \infty$, we see from (3.3) and (3.4) that

$$
\lim _{n \rightarrow \infty} \frac{1}{n} \sum_{k=1}^{n} \alpha_{k} f\left(\xi_{k}^{(n)}\right)=\alpha(R) \oint_{0}^{1} f(x) d x,
$$

which is the desired result.

4. Miscellaneous results. The following is an extension of Theorem 3.1 .

ThEOREM 4.1. Let $\Phi_{0}^{1} f(x) d x$ be convergent. Let there be a $g(x)$ with the following properties:

1. In the interval $0<x \leqq r_{2}$ where $0<r \leqq 1, g(x)$ is positive and tends steadily to $\infty$ as $x$ tends to zero.

2. $\oint_{0}^{r} g(x) d x$ is convergent.

Let $|f(x)| \leqq g(x), 0<x \leqq r$. Let $1 / M n \leqq \xi_{1}^{(n)} \leqq 1 / n$ ( $M \geqq 1$ and fixed), and $(k-1) / n \leqq \xi_{z}^{(n)} \leqq k / n(k=2,3, \cdots, n)$. Let the pattern $P$ be characterized by the dyadic number

$$
t=0 . \alpha_{1} \alpha_{2} \alpha_{3} \cdots \alpha_{n} \cdots(2),
$$

such that 


$$
\lim _{n \rightarrow \infty} \frac{1}{n} \sum_{k=1}^{n} \alpha_{k}=\alpha
$$

Then,

$$
(P) \oint_{0}^{1} f(x) d x \equiv \lim _{n \rightarrow \infty} \frac{1}{n} \sum_{k=1}^{n} \alpha_{k} f\left(\xi_{k}^{(n)}\right)=\alpha \oint_{0}^{1} f(x) d x .
$$

Since the proof is similar to that of Theorem 3.1, it will be omitted. It is of interest to note that if one chooses

$$
f(x)=\frac{1}{x} \cos \frac{1}{x}
$$

then $\oint_{0}^{1} f(x) d x$ converges, but

$$
\lim _{n \rightarrow \infty} \frac{1}{n} \sum_{k=1}^{n} f\left(\xi_{k}^{(n)}\right)
$$

fails to exist.

Define

$$
\begin{aligned}
& A_{n}=\frac{1}{n} f\left(\frac{1}{M n}\right)+\frac{1}{n} \sum_{k=2}^{n} f\left(\frac{k-1}{n}\right) \quad(M \geqq 1 \text { and fixed }), \\
& B_{n}=\frac{1}{n} \sum_{k=1}^{n} f\left(\frac{k}{n}\right) .
\end{aligned}
$$

Then $A_{n}-B_{n}=(1 / n)\{f(1 / M n)-f(1)\}$. If (4.1) exists,

$$
\lim _{n \rightarrow \infty} M \frac{1}{M n} f\left(\frac{1}{M n}\right)=0,
$$

which is certainly not the case when $f(x)=(1 / x) \cos (1 / x)$.

\section{BIBLIOGRAPHY}

1. R. E. Carr and J. D. Hill, Pattern integration, Proceedings of the American Mathematical Society vol. 2 (1951) pp. 242-245.

2. T. J. I'a Bromwich and G. H. Hardy, The definition of an infinite integral as the limit of a finite or infinite series, The Quarterly Journal of Pure and Applied Mathematics vol. 39 (1907-1908) pp. 222-240.

3. A. Wintner, The sum formula of Euler-Maclaurin and the inversions of Fourier and Möbius, Amer. J. Math. vol. 69 (1947) pp. 685-708.

Michigan State College 